\title{
An Insight into the Use of Smart Antennas in Mobile Cellular Networks
}

\author{
Carmen B. Rodríguez-Estrello and Felipe A. Cruz Pérez \\ Electric Engineering Department, CINVESTAV-IPN \\ Mexico
}

\section{Introduction}

3G and 4G cellular networks are designed to provide mobile broadband access offering high quality of service as well as high spectral efficiency ${ }^{1}$. The main two candidates for $4 \mathrm{G}$ systems are WiMAX and LTE. While in details WiMAX and LTE are different, there are many concepts, features, and capabilities commonly used in both systems to meet the requirements and expectations for $4 \mathrm{G}$ cellular networks. For instance, at the physical layer both technologies use Orthogonal Frequency Division Multiple Access (OFDMA) as the multiple access scheme together with space time processing (STP) and link adaptation techniques (LA)

In particular, Space Time Processing has become one of the most studied technologies because it provides solutions to ever increasing interference or limited bandwidth (Van Rooyen, 2002), (Paulraj \& Papadias, 1997). STP implies the signal processing performed on a system consisting of several antenna elements in order to exploit both the spatial (space) and temporal (time) dimensions of the radio channel. STP techniques can be applied at the transmitter, the receiver or both. When STP is applied at only one end of the link, Smart Antenna (SA) techniques are used. If STP is applied at both the transmitter and the receiver, multiple-input, multiple-output (MIMO) techniques are used. Both technologies have emerged as a wide area of research and development in wireless communications, promising to solve the traffic capacity bottlenecks in $4 \mathrm{G}$ broadband wireless access networks (Paulraj \& Papadias, 1997).

MIMO techniques and their application in wireless communication systems have been extensively studied (Ball et. Al, 2009), (Kusume et. Al, 2010), (Phasouliotis \& So, 2009), (Nishimori et. al, 2006), (Chiani et. al, 2010), (Seki \& Tsutsui, 2007), (Hemrungrote et. al, 2010), (Gowrishankar et. al, 2005), (Jingming-Wang \& Daneshrad, 2010); however, critical aspects of using SA techniques in cellular networks remain fragmental (Alexiou et. al, 2007). In particular those aspects related with the influence of users' mobility and radio environment at system level in SA systems which use Spatial Division Multiple Access (SDMA) as a medium access technique.

\footnotetext{
${ }^{1}$ A measure often used to assess the efficiency of spectrum utilization is the number of voice channels per Mhz of available bandwidth per square kilometer (Hammuda, 1997). This defines the amount of traffic that can be carried and is directly related to the ultimate capacity of the network.
} 
SDMA cellular systems have gained special attention to provide the services demanded by mobile network users in 3G and 4G cellular networks, because it is considered as the most sophisticated application of smart antenna technology (Balanis, 2005) allowing the simultaneous use of any conventional channel (frequency, time slot or code) by many users within a cell by exploiting their position. However, SDMA technology has not been widely integrated into cellular systems as many as it had been predicted because SDMA introduces new challenges at the system level modeling. In particular, the design of radio resource management algorithms is an open research topic (Alexiou, A. et. al, 2007), (Toşa, 2010) Furthermore, in order to measure the performance of different radio resource management algorithms it is necessary to develop an adequate system level model because in SDMA cellular system level performance is closely affected by the constantly changing radio environment due to the users' mobility.

Thus, the objective of this chapter is to give an overview of the smart antenna technology in mobile cellular systems emphasizing those features which are related with SDMA. This chapter highlights the critical aspects of system level modeling regarding to radio resource management algorithms; in particular, users' mobility and radio environment issues are considered.

The chapter is organized as follows: In the first part an overview of smart antenna technology is given. Then, the techniques and applications of smart antennas in cellular systems are explained. After that, some commercial systems that use smart antennas are described. Afterward, the proposed model to include channel characteristics at system level is presented. Finally, the impact of users' mobility and radio environment on the system performance is evaluated.

\section{Overview of smart antenna technology}

The term smart antennas generally refers to any antenna array joint with signal processing, which can adjust or adapt its own beam pattern in order to emphasize signals of interest and to minimize interfering signals (Gross, 2005), (B. Allen and M. Ghavami, 2005). Smart Antennas can modify their radiation pattern by means of an internal feedback control while the antenna system is operating.

Smart antennas have alternatively been labeled through the years as adaptive arrays or digital beam forming arrays. The development of adaptive arrays began in the late 1950s. The term "adaptive arrays" was first coined by Van Atta (Van Atta, 1959) in 1959 to describe a self phased array. Self phased arrays reflect all incident signals back in the direction of arrival by using phase conjugation. Self phased arrays are instantaneously adaptive arrays since they essentially reflect the incident signal in a similar fashion to the classic corner reflector (Balanis, 2005).

Lately, in 1965, an adaptive sidelobe canceller was developed by Howell and Applebaum (Applebaum, 1976). This technique allows for mitigating interference, raising the signal to interference ratio (SIR). Another type of adaptive beamformer was developed by (Widrow et. al, 1967); this adaptive beamformer uses a pilot signal as a reference. It operates by forming a beam towards the wanted source(s) whilst simultaneously directing nulls towards interference sources. The beam was steered via phase shifters, which were often implemented at RF stage. This general approach to phase shifting has been referred as electronic beamsteering because the phase change is made directly at each antenna element (Gross, 2005). 
Modern smart antennas systems still continue using the previously described techniques, but taking advantage of digital signal processing. The digital processing is performed at base band frequency, instead of doing it at RF stage. More over new beamforming techniques have emerged based on digital signal processing.

Due to the characteristics of smart antennas, they were originally focused on military uses like radar. Then smart antennas have been also employed in satellite applications to reuse frequency channels in different geographic locations. Recently, smart antennas were used in fixed wireless communication systems as wireless local loop (WLL). Nowadays, smart antennas are used in mobile wireless communication systems to improve coverage, capacity and spectral efficiency (Alexiou, A. et. al, 2007), (Toşa, 2010), iBurst, (2004), (3GPP TR 25.913, 2009). In particular, in cellular systems the use of smart antennas allows lower cost deployments with cells of moderate large size.

\section{Architecture of smart antenna systems}

As it was established, modern smart antennas are antenna arrays aided by digital signal processor. Thus, a generic smart antenna consists of two major components: the antenna array and the digital signal processor as it is shown in Figure 1.

\subsection{Antenna array}

The antenna array is one of the constitutive parts of a smart antenna: an antenna array consists of $N$ identical independent antenna receivers separated in the space allocated in a geometric form. Thus, the electrical size of the complete array is greater than the electrical size of an individual element. By increasing the electrical size, highly directive radiation patterns are formed. Moreover, the multiplicity of elements allows more precise control of the radiation pattern resulting in lower sidelobes or fine pattern shaping.

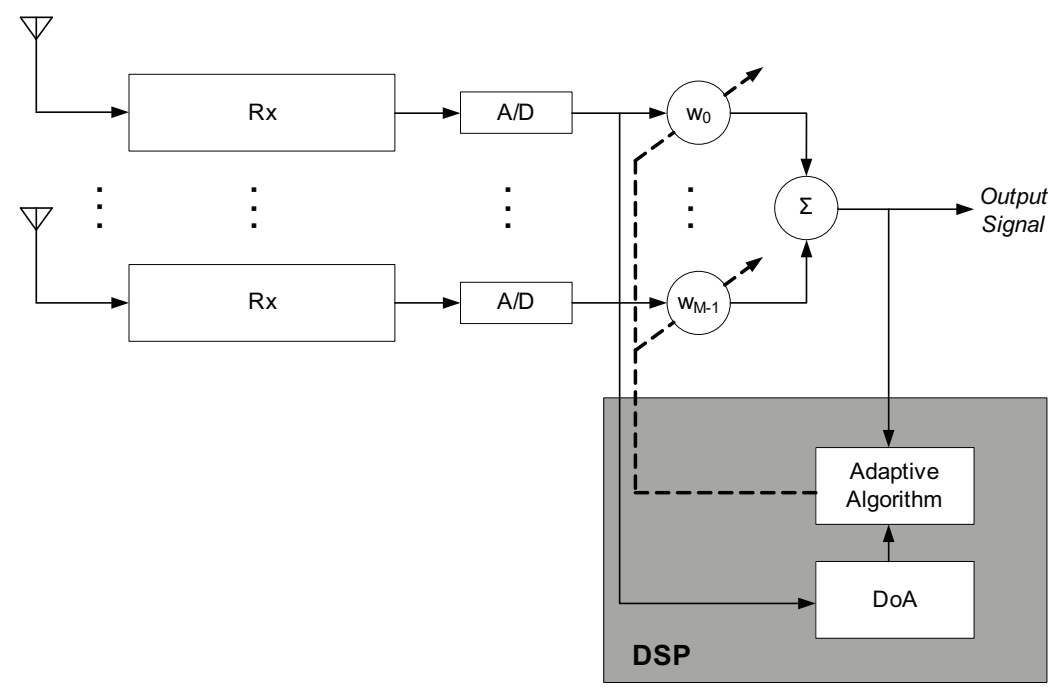

Fig. 1. A generic smart antenna system (Balanis, 20005) 
The total radiated (received) field of the array at any point in the space is the vectorial sum of radiated (received) fields by each individual antenna (Balanis, 2005). Thus, the total radiated (received) field is determined by the product of individual element pattern and the array factor. The array factor is the spatial response to the received signals and it is affected by the geometrical relation of individual elements. Consequently, in order to provide directive patterns it is necessary that the fields from the elements of the array interfere constructively in the desired directions and interfere destructively in the remaining space. In an array of identical elements, there are five parameters of control that can be used to shape the overall pattern of antenna (Balanis, 2005):

The number of the elements of the array. The number of antenna elements in the array determines the degrees of freedom (to create nulls or maxima in the beampattern). That is, an antenna array with $N$ elements allows forming $N$ nulls or maxima.

The relative displacement between elements. The correlation among radiated (received) fields of the individual elements is influenced by the relative displacement between them. The displacement between elements has a direct relation with the physical and electrical array size. Displacement is measured in terms of wavelength because the response of the array is closely related with the operation frequency.

The separation between elements is also associated with a particular application. For instance, systems which use diversity need antenna arrays with relative displacements that ensure uncorrelated fading of the signals (more than one wave length). On the contrary, beamforming applications usually require relative displacements of less than a half wavelength. Relative displacement between elements is usually restricted by the available space for the antenna array.

The geometrical configuration of the overall array. The physical shape obtained with the allocation of the elements of the array is known as the geometry of the array. Location of the individual elements can vary widely, but the most common configurations are along a straight line, around a circle or in a planar way. The geometry of the antenna determines the relationship between the radiated fields of the individual elements. Thus, many important characteristics of the beampattern hang on the geometry. For example, linear and planar arrays generate grating lobes ${ }^{2}$ if the relative displacement between elements is more than a half wavelength. Thus, linear arrays are typically used if sectored coverage is required. While circular or hexagonal arrays are used to provide 360 degrees continuous coverage.

The excitation (amplitude and phase) of the individual elements. Received (radiated) signals are weighted at each element and they are sum to form the beam pattern. Weights are in general complex in order to determine amplitude and time delay (phase) of the signal that feeds each antenna element. The weights can be approached in a fixed or adaptive way.

The relative pattern of the individual elements. Relative pattern is the radiated pattern of each element when it is not arranged in an array. Even though, it is possible to use any antenna as element in the array, individual elements used in arrays are generally half wavelength dipoles $^{3}$, to ensure that the radiation pattern of the overall array is completely determined by the geometry.

\footnotetext{
${ }^{2}$ Grating lobes are defined as: lobes, other than the main lobe, produced by an antenna array when the element spacing in the same plane is sufficiently large to permit the in-phase of radiated fields in more than one direction. (Balanis, 2005)

${ }^{3}$ Half wavelength dipoles present omnidirectional patterns. (Balanis, 2005)
} 


\subsection{Signal processing}

The purpose of the signal processing stage is to adapt the beampattern to the radio environment conditions. This one is because signal processing has been used together with antenna arrays to act as spatial filters. Such filters can self-adjust to the characteristics of an incoming signal without external intervention, just as a closed loop control system. Thus, signal processing combined with antenna arrays produce a directive beam that can be repositioned (scanned) electronically by varying the excitation of the individual elements. Then, the objectives of the signal processing stage are (Balanis, 2005):

Estimate the direction of arrival (DoA) of all impinging signals. DoA estimation techniques can be categorized on the basis of the data analysis and implementation into four different areas (Liberti \& Rappaport, 1999) (Krim \& Viberg, 1996):

- Conventional methods, Conventional methods are based on beamforming and null steering. In this technique, DoA is determined tracking peaks by means of an exhaustive search on all possible directions. Examples of conventional methods are delay and sum, and Capon's minimum variance method.

- Subspace-based methods. Different from conventional methods, subspace methods exploit the structure of the received data; in particular the covariance matrix. This one results in a great improvement in resolution. Examples of these methods are MUltiple SIgnal Clasification (MUSIC) and the Estimation of signal parameters via rotational invariance technique (ESPIRIT) (Schmidt, 1979).

- Parametric methods. These methods are used in scenarios where the involved signals are highly correlated, or even coherent. Even though, parametric methods increase the efficiency and robustness of DoA, parametric estimation methods are computationally more complex than conventional and subspace methods. However, for Uniform Linear Arrays (ULA) there are a number of less demanding algorithms (Krim \& Viberg, 1996). Maximum likelihood (deterministic and stochastic) algorithms are the most frequently used. These algorithms are based on finding the optimum data processing solution for the case of $J$ unknown signals and $K$ sensors $(K>J)$ with only additive white noise errors (Schweppe,1968) (Ziskind \& Wax, 1988)

- Integrated methods, which combine two or more methods. There are many combined methods which try to get the advantages of both methods while minimizing disadvantages. However, most of these methods are computationally very complex. (Parra et. al, 1995).

Calculate the appropriate weights to steer the maximum radiation of the antenna pattern toward the Signals of Interest (SoI) and to place nulls toward the Signals of No Interest (SNoI). The algorithms used to form a beampattern could be categorized in two kinds: those which are DoA-based and those which uses a reference signal or a training sequence (Balanis, 2005).

DoA-based beamforming algorithms. The information supplied by the DoA algorithm is processed by means of an adaptive algorithm to ideally steer the maximum radiation of the antenna pattern toward the SoI and place nulls in the pattern toward the SNoI.

Reference training beamforming algorithm. In these techniques, an excitation vector that minimizes a cost function is determined. The cost function is related to a performance measure and it is inversely associated to the quality of the signal at the array output. The most commonly used performance measures are Minimum Mean Square Error (MMSE), Maximum Signal to Noise Radio (MSNR) and Minimum Variance (MV) (Litva, 1996). If the cost function is minimized, then, the quality of the signal is maximized (Liberti \& Rappaport, 1999). In order to minimize the cost function, reference training algorithms are 
used. These algorithms require solving a linear system of equations based on the information of training sequences. If the radio environment is stationary, the arrival angles of desired and undesired signals do not change. Consequently, weights are easily computed. However, if the radio environment is continuously changing, weights are needed to be computed with adaptive methods and they become computationally extremely demanding (Balanis, 2005). The most common used adaptive algorithms are Least Mean Squares (LMS), Sample Matrix Inversion (SMI), and Recursive Least Squares (RLS) (Gross, 2005).

Blind beamforming algorithms. These techniques exploit the characteristics of the signal, such as autocorrelation, when no reference signal is available. Blind beamforming techniques can be used as adaptive methods to constantly calculate the appropriate weights to steer the beam. An example of blind beamforming algorithm is the family of Constant Modulus (CM) algorithms, which takes advantage of the constant amplitude of phase-modulated signals (Gross, 2005). Decision-Directed algorithm and cyclostationary algorithms are other examples of blind beamforming (Litva, 1996).

\section{Smart antenna systems categorization}

Smart antennas systems can be categorized by considering the adaptation technique and their application to cellular systems. The adaptation technique refers to the capacity of the algorithm to track the user and to eliminate undesired signals and the application refers to the objective of using smart antennas in cellular systems.

\subsection{Smart antenna adaptation techniques}

Smart antennas generally encompass both switched and beamformed adaptive systems. Switched or fixed beam is the simplest one and it refers to the arrangement in which a finite number of predefined radiation patterns are formed in fixed directions. While the adaptive array approach denotes the capability of smart antennas to dynamically adjust the radiation pattern to improve the performance of the system according to a certain performance metric. Switched beam. As an extension of sectorised concepts, the objective of switched beam systems is to form several available fixed beam patterns. In switched beam systems, the maxima of the fixed beampatterns are selected to ensure a uniform coverage of a region in the space. Normally, directions of the maximums are in equal angular increment.

Switched beam is technologically the simplest technique and can be implemented by using a number of fixed, independent, directional antennas or virtually with an antenna array and an analogue beamformer such as Butler matrix or roman lens (Butler, 1961). The operation of Butler matrix can be likened to a Fast Fourier Transform (FFT) and yields $M$ mutually orthogonal beams. The orthogonality of the beams is defined by the angle minima of one beam pattern corresponding with the main beam angle of all of the other beams (Butler, 1961). A similar technique called grid of beams $(\mathrm{GoB})$ can be used with digital beamforming systems which selects the best weights from a stored set (Tsoulos, 1999). This technique leads to a more complex implementation due to the drawbacks associated with digital beamforming.

In switched beam systems, the decision of which beam serves a specific user is made based upon the requirements of the system. For instance, one criterion which can be applied to select a beam is to maximize Signal to Interference Ratio (SIR); another criterion is to maximize the Received Signal Strength Indicator (RSSI), which consist in select the beam which provides the strongest signal. 
Several works have studied switched beam systems at link and at system level (Mailloux, 1994), (Hansen, 1998), (Pattan, 2000). Many of these works have studied the switched beam systems at link level analyzing SNR and Bit Error Rate performance (Hu \& Zhu, 2002), (Nasri et.al, 2008) (Ngamjanyaporn, 2005) (Lei et. al, 2005). In (Ho et. al, 1998) (Peng \& Wang, 2005) the performance and feasibility at system level of switched beam systems has been investigated in terms of blocking probability.

Even though, switched beam systems provides an increment in the capacity of cellular systems, as the issue of trunking efficiency has become more pronounced, focus has recently shifted to more advance fully adaptive techniques.

Beamformed adaptive systems allow the antenna to steer the beam to any direction of interest while simultaneously nulling interfering signals. Adaptive array antenna systems continually monitor their coverage areas attempting to adapt to their changing radio environment which consists of mobile user and mobile interferers. In the simplest scenario (one users and no interferers) the systems adapts to the user's motion producing and effective antenna pattern that follows the user always providing maximum gain in the user's direction.

In adaptive systems, pattern optimization is done by real time active weighting of the received signal and can adapt to changes in the radio environment. Although in principle it is possible to adapt transmit patterns to optimize the transmission subject to some received signal or noise distribution, this is seldom done except for the formation of retrodirective beams which automatically transmit in the direction of the received signal or pilot tone (Balanis, 2005)

The switched beam technique is more attractive when compared to the adaptive null steering because with switched beam, no complicated multi-beam beamforming is needed and no significant changes to the existing cellular systems are required (Peng \& Wang, 2005) (Shim \& Choi, 1998).

\subsection{Smart antenna applications in cellular systems (HSR, SFIR, SDMA)}

The usages of smart antennas in cellular systems are focused on three different objectives: increasing coverage as a High Sensitivity Reception (HSR), reducing interference as spatial filters -Spatial Filter Interference Reduction- (SFIR), and spatially reusing basic radio resources (frequency bands, time slots, orthogonal codes, chunks) providing another way of multiple access -Spatial Division Multiple Access- (SDMA). These applications have been also considered as the stages of introduction of smart antenna technology in the evolution of cellular systems (Boukalov \& Haggman, 2000).

High Sensitivity Reception (HSR). Smart antennas were originally used to provide range extension through the inherent directional gain obtained from using an array. The additional directional gain, $D$ provided by an $M$ element array is approximated by:

$$
D \simeq 10 \log _{10} M
$$

This application is useful for rural cells which are required to cover larger areas than those in urban environments.

Spatial Filtering Interference Rejection (SFIR). Smart Antennas are also applied to achieve spatial filtering, enhancing the signal strength (beamforming) and weakening the interference power (nulling) in Wireless Communication Systems. Network capacity is increased by controlling the interference level received from other users and base stations. This operating mode is referred to as spatial filtering interference rejection (SFIR). 
SFIR reduces susceptibility to multipath effects since spatial filtering reduces the channel delay spread. This is because signals arriving at angles outside of the main beam are attenuated. These signals will have longer path lengths and would normally contribute to the longer delays of the channel impulse response. The consequence of reducing delay spread is that equalization techniques are no longer required therefore simplifying the receiver design. Moreover, channel reuse patterns in cellular systems can be significantly tighter because the average interference resulting from co-channel signals in other cells is markedly reduced.

Spatial Division Multiple Access (SDMA). The most promising mode of smart antennas in cellular systems is referred to as space division multiple access (SDMA) where smart antennas are used to separate signals, allowing different subscribers to share the same resource provided their signals are spatially separable at the base station. This mode of operation allows multiple users to operate on the same slot, frequency and in the case of CDMA systems, the same code within a cell. The net result of the adaptive process is that SDMA systems can create a number of two way spatial channels on a single conventional channel (frequency, time, code). SDMA technology is not restricted to any particular modulation or air interface protocol and is compatible with all currently air interfaces deployed at this time.

Modern wireless communications systems deploy antenna arrays in SDMA configuration. Here a base station communicates with several active users by directing the beam towards them and it nulls users which cause interference. This has two beneficial effects: first the target users receive more power compared to the omnidirectional case and second the interference to the adjacent cells is decreased because only very selected directions are targeted.

\section{Commercial smart antennas cellular systems}

Although smart antennas have been a hot research topic in the last two decades, and smart antenna techniques have been proposed in 3G and 4G cellular systems (ITU-R M.1801, 2007), (3GPP TR 25.913, 2009), (Hoymann, 2006) as one of the leading technologies for achieving high spectral efficiency ${ }^{4}$ by reusing basic radio resources, smart antenna systems are slowly becoming commercially available. The main reasons which explain why smart antenna systems have not been deployed completely are stated in (Alexiou et.al., 2007), (Kaiser, 2005), (Rajal, Dec 2005), (G. Okamoto, 2003). The conclusions are that the speed of DSP is not enough to real - time process the needed algorithms and the algorithms are computationally very demanding. Moreover the additional cost of using smart antenna at current systems is not sustainable.

\footnotetext{
${ }^{4}$ Spectral efficiency measures the ability of a wireless system to deliver information, within a given amount of radio spectrum. In cellular radio systems, spectral efficiency is measured in bits/second/Hertz/sector (bps/Hz/sector). Factors that contribute to the spectral efficiency include the modulation formats, "overhead" due to the signaling, multiple access method, etc. The reason to reference of the spatial dimension (per sector) is the self interference generated in the network, requiring the operator to allocate frequencies in blocks that are separated in space by one or more cells. This separation is represented by a reuse factor, where a lower number is representative of a more efficient system.
} 
Some efforts have been made in order to standardize the use of smart antennas in wireless communication networks. Several standardization organizations such the Alliance of Telecommunications Industry Solutions (ATIS), the International Telecommunication Union (ITU), and the Institute of Electric and Electronic Engineers have standardized the use of smart antennas in wireless systems. Furthermore, some field tests for MIMO and SA technologies have been developed (TSUNAMI) (Tsoulos \& Mark Beach, 1997). However, currently, only a few companies have successfully commercialized smart antenna systems for cellular base stations.

The first commercial system that uses smart antennas was iBurst. iBurst is a mobile broadband wireless access system that was first developed by ArrayComm, and subsequently adopted as the High Capacity - Spatial Division Multiple Access (HC-SDMA) radio interface standard (ATIS-0700004-2005). ITU also includes this system in ITU-R M.1678 and ITU-R M.1801.

ITU-R M.1678 recommendation addresses the use of adaptive antenna technology in the mobile service with the objective to improve spectrum efficiency significantly, improve the ability of mobile systems to coexist and facilitate cross-border and adjacent band sharing, and facilitate the deployment of new wireless networks, including broadband wireless access and radio local area network systems.

ITU-R M.1801 recommendation defines specific standards for broadband wireless access in the mobile service at radio interface. These specific standards are composed of common specifications developed by standards development organizations (SDOs). Using this Recommendation, manufacturers and operators should be able to determine the most suitable standards for their needs. These standards support a wide range of applications in urban, suburban and rural areas for both generic broadband internet data and real-time data, including applications such as voice and videoconferencing. The commercial name of this recommendation is High Capacity - Spatial Division Multiple Access (HC-SDMA).

In January 2006, the IEEE 802.20 Mobile Broadband Wireless Access Working Group adopted a technology proposal that includes the use of the HC-SDMA standard for the $625 \mathrm{kHz}$ Multi-Carrier Time Division Duplex (TDD) mode of the future IEEE 802.20 standard. Moreover the recommendation IEEE 802.16 proposes the use of smart antennas in WiMAX system and 3GPP group suggests also the use of smart antennas in LTE.

iBurst (HC-SDMA) is a wireless broadband technology developed by ArrayComm. The main objective of the iBurst system is to optimize the use of the available bandwidth with the help of smart antennas. Arraycomm and Kyocera are the main providers of the equipment for this technology. HC-SDMA offers up to $20 \mathrm{Mbps}$ of aggregate usable IPtraffic capacity per sector in each $5 \mathrm{MHz}$ TDD allocation and supports both fixed and fully mobile broadband users. As the standard's name implies, the key to the system's capacity is the spatial processing and interference management software, supporting up to 3 SDMA channels on each physical carrier in each cell. HC-SDMA works with TDD/TDMA/SDMA, $625 \mathrm{kHz}$ channel spacing (iBurst, 2004).

Although, iBurst is an in-use commercial system, it actually experiences some drawbacks when it deals with users' mobility in the commercial implementation. Actually, ArrayComm reports in a white paper (iBurst, 2004), that "SDMA is most useful to operators with high capacity requirements, more limited spectrum, and tight constraints on client device costs and complexity. However, the effectiveness of SDMA declines gradually with increasing subscriber mobility".

ArrayComm has also implemented HSR and SFIR applications. Their solution for increased gain and interference management for GSM infrastructure includes products, designed to 
improve frequency re-use and overall network capacity. Moreover, ArrayComm's ranging extension solution for WiMAX provides a $\sim 6 \mathrm{~dB}$ improvement in ranging channel link budget. This enables successful and practical use of all the traffic-channel range gains identified above.

Regarding to the Smart Antenna techniques, ArrayComm reports in (iBurst WP, 2004) that Beam switching technology has seen virtually no commercial use. Drawbacks include high sensitivity to the subscriber's location within the beam and interference from users outside the beam's primary target. Moreover, they consider that beam steering algorithms have also not seen successful use outside the laboratory. The radio environment in the world of commercial services is filled with multipath and scattering effects and non-line-of-site conditions that prevent techniques based on degree-of-arrival calculations from delivering useful results in most circumstances. Thus, more sophisticated algorithms are required as well as faster digital signal processors.

Other company that is working on smart antenna applications is Alvarion (SentieM WP, 2008). Alvarion's SentieM Mobile WiMAX is not currently available; however, it is on development. Alvarion's future SentieM Mobile WiMAX technology uses Spatial Division Multiple Access (SDMA) technology which provides the ability to use the same frequency (beam) at the same time for different users. This solution is unique in its ability to select the right user at the right time for the frequency sharing to work. Alvarion reports that "SentieM's SDMA solution will not require any information from the end-user terminal, avoiding the need for a complicated integration process with the subscriber device".

Ericsson has also has conducted extensive research and development of advanced basestation antennas for mobile communication (Derneryd \& Johannisson, 1999). Their work comprises both adaptive and active antenna systems. With the introduction of active antenna products, such as Maxite products, small-sized base station units with high levels of equivalent radiated power (ERP) and low power consumption can be used.

Ericsson has also developed an HSR system which consists of two-dimensional antenna arrays for adaptive base-station systems. These arrays, which are developed for systems based on GSM and TDMA (IS 136) standards, work in the 900, 1800 or $1900 \mathrm{MHz}$ frequency bands. Together with Mannesmann Mobilfunk GmbH (GSM) and AT\&T Wireless Services (TDMA), Ericsson has conducted field trials in live networks to evaluate the performance of the adaptive systems. The results show that adaptive antenna systems increase capacity in $20 \%$. The adaptive array antenna transmits and receives radio-frequency signals in directed narrow beams in the base of Butler matrices to produce horizontal beamforming networks. As a consequence of the use of fixed beamforming networks, besides increased capacity, the increase in antenna gain may also be exploited to offer greater coverage.

\section{System-level model for mobile SDMA cellular systems}

Although the use of smart antennas in cellular systems lead to a capacity increase, the deployment of smart antennas implies a more complicated design at radio stage and new radio resource management algorithms designed specifically for this kind of systems (Boukalov, 2000). In particular, in SDMA cellular systems, intra- and inter-cell co-channel interference is increased because of the intra-cell cochannel reuse. As a result, is not always possible to replicate basic channels ${ }^{5}$ in the admission process. In addition, the effect of intra-

${ }^{5}$ Basic channels are considered time slots, frequency carriers and codes 
cell interference becomes worst as the users' mobility increases. Consequently, users' SIR could be severely degraded due to users' mobility.

Previous published works have mathematically analyzed SDMA at system level by means of a multidimensional Markov model (Galvan-Tejada \& Gardiner, 1999), (Galvan-Tejada \& Gardiner, 2001), (Shuangmei et. al 2004); however, users' mobility together with co-channel interference due to the replicated channels within cells has not been considered. Thus, only new call blocking probability is calculated and call forced termination probability is disregarded. On the other hand, most of the studies addressing the impact of users' mobility at the system level performance of SDMA cellular networks have been done through discrete event computer simulations (Pabst et. al, 2007), (Czylwik et. al, 2001), (Cardieri \& Rappaport, 2001). However, they are based only on geometrical considerations (i.e., hexagonal/circular shaped cells, linear users' movement, ideal beam patterns) while current co-channel interference conditions experienced by users are ignored at all. Just a few works have dealt with mobility in an analytical way (Tangeman, 1994), (Liu, 2004), (Liu, 2005) and none of the previous works have treated mobility and co-channel interference together.

In order to include users' mobility and the effect of interference at system level, it is necessary to develop and adequate teletraffic model. In (Rodríguez-Estrello \& Cruz-Pérez, 2009) a system level analytical model which includes not only mobility but also co-channel interference for SDMA systems, Thus, in this section, the model proposed in (RodríguezEstrello \& Cruz-Pérez, 2009) is taken as a basis to analyze SDMA system's performance.

On the other hand, in order to evaluate the effects of mobility, the generalized mobility model proposed in (Zonoozi \& Dassanayake, 1997) is used for random user mobility characterization due to its simplicity and versatility to represent several scenarios. The model in (Zonoozi \& Dassanayake, 1997) is characterized by the parameter $\alpha$ that limits the range of maximum variation of the future moving direction relative to the current one.

\subsection{Network topology}

A real time (i.e., conversational) service homogeneous mobile multi-cellular system with smart antennas located at the center of cells is assumed. Figure 2 shows the network topology. SDMA is used as a multiple access scheme in conjunction with a basic multiple access scheme (TDMA, CDMA, OFDMA). Thus, two or more users could share a basic channel within a cell (intra-cell reuse); however, resources could be 'replicated' only if the SIR is above a threshold in both channels.

\subsection{Proposed Model to Include Co-channel Interference}

The effect of co-channel interference is captured through the system level model proposed in (Rodríguez-Estrello \& Cruz-Pérez, 2009) by introducing two parameters that depends on the mobility and radio environment:

The acceptance probability. The acceptance probability is the probability that a basic resource could be replicated in the admission process. This probability reflects the probability that the SIR is above a given threshold. Notice that this probability depend on how many times the basic resource is replicated.

Poisson call interferential process. The proposed model in (Rodríguez-Estrello \& Cruz-Pérez, 2009) is based on the physical process in which a call could be involved: after a new call or a handoff attempt is accepted by a base station (BS), if the call is served by a replicated channel, the link condition could become degraded mainly due to the intra-cell co-channel 


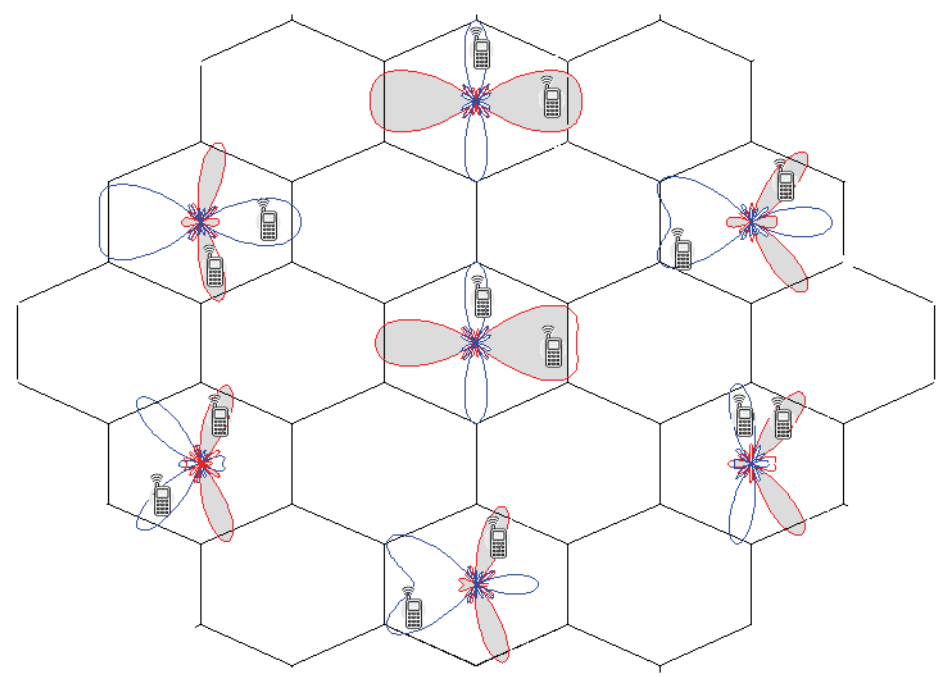

Fig. 2. Network Topology

interference causing a fall on the SIR of involved users and forced to terminate the call due to link unreliability. The period of time between the instant the call is originated and the moment the call is forced to terminate due to link unreliability is called "beam overlapping time". Thus, the proposed interferential process is a Poisson process which reflects the effect of link conditions and it has associated the beam overlapping time.

Beam overlapping time strongly depends on how many users share the same channel. Therefore, in general, the more users sharing a channel, the shorter beam overlapping time. Physically, beam overlapping time represents the period of time in which the users' SIR would be degraded due to the co-channel interference under the assumption that both cell dwell time and the unencumbered service time are of infinite duration.

\subsection{Call Admission Control (CAC)}

In SDMA cellular systems, call admission control refers the replicating order of basic radio resources. Two replication policies are possible:

Duplicate at last. (DL) Allocate basic channels one by one; once all $\mathrm{N}$ basic channels have been assigned, allocate the replicas of the basic channels (if the channel conditions are good enough to permit replicate).

Duplicate at first. (DF) Allocate basic channels and replicate them even if the all basic channels have not been previously allocated. If it is not possible to assign a replicated channel, allocate next primary channel.

\subsection{Teletraffic model}

\subsubsection{General guidelines}

For the mathematical analysis, the general guidelines of the model presented in (Lin et. Al, 1994) are adopted here to cast the system here considered in the framework of birth and death processes: 
1. A homogeneous multi-cellular system with smart antennas located at the centre of each cell is assumed.

2. Each cell has a maximum number of basic radio resources $C$, each one of these basic channels could be replicated up to $R$ times (intra-cell reuse factor) due to the SDMA capability.

3. The unencumbered call duration and cell dwell time are assumed to be negative exponentially distributed random variables with mean $1 / \mu$ and $1 / \eta$, respectively.

4. The probability that $i$ channels can be replicated $k$ times in the admission process is denoted by $p_{i, k}$. These probabilities have been extensively studied and characterized in the literature by means of mathematical analysis (Galvan-Tejada \& Gardiner, 1999), (Galvan-Tejada \& Gardiner, 2001), (Shuangmei et. al 2004), and it has been demonstrated that they can be expressed in terms of the probabilities that one channel could be replicated $k$ times.

$$
p_{i, k}=1-\left(1-p_{1, k}\right)^{i}
$$

5. It is considered that no reassignment nor prioritization scheme for handoff calls or new calls is used6.

6. New call arrival process follows a Poisson process with mean arrival rate $\lambda_{\mathrm{n}}$ per cell.

7. Handoff call arrival process to every cell is also considered to be a Poisson process with mean arrival rate $\lambda_{h}$. The inter-cell handoff arrival rate is iteratively calculated using the method described in (Lin et. al, 1994) ${ }^{7}$.

8. Call interferential process is considered to be a Poisson process with mean arrival rate $\gamma_{i}$, where $i$ is the number of users sharing the same channel within a cell. Characterization of beam overlapping time has been previously reported in (RodríguezEstrello et. al, 2009)

\subsubsection{Queuing formulation}

As a homogenous case is assumed, the overall system performance can be analyzed by focusing on only one given cell and considering that its neighboring cells exhibit identical statistical behavior. Let us denote the state of a given cell as $\mathbf{K}=\left[k_{1}, k_{2}, \ldots k_{R}\right]$, where $k_{\mathrm{j}}$ (for $i=$ $1,2, \ldots, R)$ represents the number of active mobiles in the $i$-th level of repetition ${ }^{8} ; i=1$ represents the case in which only basic resources has been used, $i=2$ represents the level in which two users are sharing one basic resource and so on. Thus, states in the system must accomplish with $k_{1} \geq k_{2} \geq \ldots \geq k_{R}$.

Multidimensional birth and death process is analyzed by "rate out equals rate in" equations (Cooper, 1990). The vector $\mathbf{e}_{i}$ is a unity vector of the same dimension of $\mathbf{K}$ and a "one" in the

\footnotetext{
${ }^{6}$ Considering a channel reservation scheme for handoff calls would bias the performance of the analyzed system and would not allow for the assessment of its "pure" performance. However, the system level performance evaluation of mobile cellular networks simultaneously considering cochannel interference, resource insufficiency and resource management schemes is desirable and can be easily achieved.

${ }^{7}$ Although handoff call arrival process generated by a single cell is clearly not Poissonian, the combined process from the six different neighboring cells can be adequately approximated by a Poisson process (Cheblus and W. Ludwin, 1995)

${ }^{8}$ Repetition level means the number of times that a basic resource is used within a cell.
} 
$i$-th position. System steady state probabilities are denoted by vector п. The equilibrium state equations are (Cooper, 1990):

$$
\pi_{\mathbf{K}}=\frac{\sum_{i=1}^{R} a_{i \mathbf{K}-\mathbf{e}_{i}} \pi_{\mathbf{K}-\mathbf{e}_{i}}+\sum_{i=1}^{R} b_{i \mathbf{K}+\mathbf{e}_{i}} \pi_{\mathbf{K}+\mathbf{e}_{i}}}{\sum_{i=1}^{R} a_{i \mathbf{K}}+\sum_{i=1}^{R} b_{i \mathbf{K}}}
$$

for $\mathbf{K}=\left[k_{1}, k_{2}, \ldots k_{R}\right]$ such that $\mathbf{K} \in \Omega$. Where $\Omega$ is the valid state space, defined by:

$$
\Omega=\left\{0 \leq k_{i} \leq C, k_{1} \geq k_{2} \geq \ldots \geq k_{R} \text { for } i=1 \ldots R\right\}
$$

When duplicate at first policy (DF) is used, new call arrival for mobiles that will generate a transition from $\mathbf{K}$ to $\mathbf{K}+\mathbf{e}_{i}$ (where $i=\{1,2,3, \ldots, R\}$ ) given by:

$$
a_{i \mathbf{K}}=\left\{\prod_{j=0}^{R-i-1}\left(1-p_{\left(k_{R-j-1}-k_{R-j}\right),(R-j)}\right) p_{\left(k_{i-1}-k_{i}\right),(i)}\left(\lambda+\lambda_{h}\right) \text { for } 0 \leq k_{i} \leq C, k_{1} \geq k_{2} \geq \ldots k_{R}\right.
$$

When duplicate at last policy (DL) is used, new call arrival for mobiles that will generate a transition from $\mathbf{K}$ to $\mathbf{K}+\mathbf{e}_{i}$ (where $i=\{1,2,3, \ldots, R\}$ ) given by:

$$
a_{i \mathbf{K}}=\left\{\prod_{j=1}^{i-1}\left(1-p_{\left(k_{j-1}-k_{j}\right), j}\right) p_{\left(k_{i-1}-k_{i}\right),(i)}\left(\lambda+\lambda_{h}\right) \text { for } 0 \leq k_{i} \leq C, k_{1} \geq k_{2} \geq \ldots k_{R}\right.
$$

Notice that if $i=1, k_{-1}=C$.

Call departure rate for users that will generate a transition from $\mathbf{K}$ to $\mathbf{K}-\mathbf{e}_{i}$ (where $i=$ $\{1,2,3 \ldots, R\})$ is given by:

$$
b_{i \mathbf{K}}=\left\{i\left(k_{i}-k_{i+1}\right)\left(\mu+\eta+\gamma_{i}\right) \text { for } 0 \leq k_{i} \leq C, k_{1} \geq k_{2} \geq \ldots k_{R}\right.
$$

Notice that if $i=R, k_{R+1}=0$. The departure rate includes the interferential rate $\gamma_{i}$ depending on the number of mobiles using replicated channels within the analyzed cell9.

Together with the normalization condition:

$$
\sum_{\{\mathbf{K} \in \Omega\}} \pi_{\mathbf{K}}=1
$$

The corresponding steady state probabilities are calculated by using the Gauss-Seidel method (Cooper, 1990).

As an example, Figure 3 shows a state transition diagram for an SDMA system that uses Duplicate at First policy with $C$ basic resources that can be replicated twice.

\footnotetext{
${ }^{9}$ This rate is strongly affected by the parameters involved in the beam forming such as number of elements in the antenna, geometry of the array, beam forming algorithm, separation between elements in the array as well as users' mobility conditions.
} 


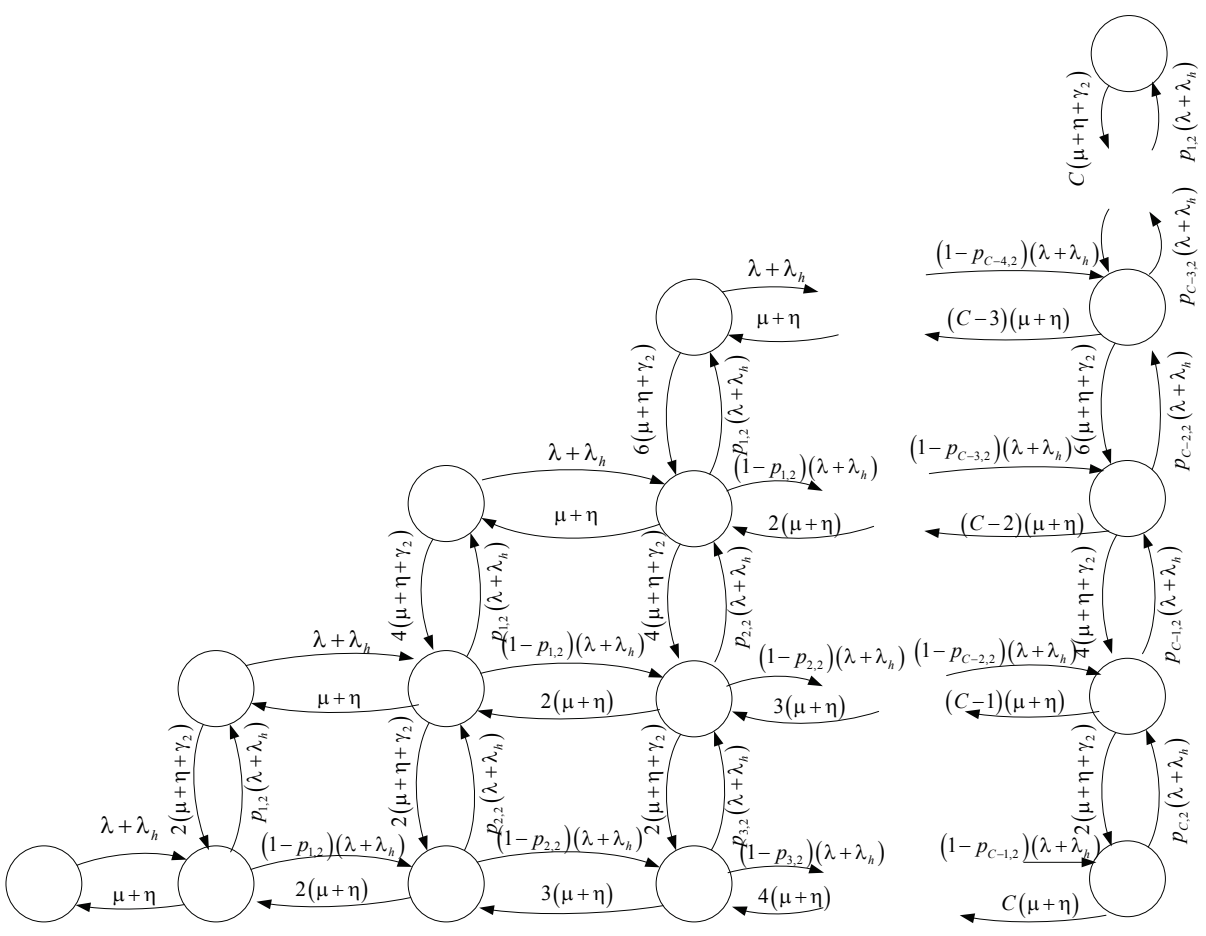

Fig. 3. SDMA system with Duplicate at First Policy.

\subsubsection{QoS metrics}

In this section, closed mathematical expressions for the most important QoS metrics are found.

Blocking probability / Handoff failure probability.

New calls are blocked with probability:

$$
P_{b}=\sum_{\left\{\mathbf{K} \in \Omega ; k_{1}=C\right\}} \prod_{j=0}^{R-1}\left(1-p_{\left(k_{R-j-1}-k_{R-j}\right),(R-j)}\right) \pi_{\mathbf{K}}
$$

Handoff probability

Handoff probability is defined as the probability that a call requires a handoff. Mathematically it is expressed as the probability that the random variable associated with the residence time $\left(\mathbf{X}_{\mathbf{r}}\right)$ is less than the minimum of the random variables associated to the service time $\left(\mathbf{X}_{\mathbf{s}}\right)$ and the beam overlapping time $\left(\mathbf{X}_{\mathbf{o i}}\right)$; that is,

$$
\mathbf{P}_{H i}=\mathrm{P}\left\{\mathbf{X}_{\mathbf{r}}<\min \left(\mathbf{X}_{\mathbf{s}}, \mathbf{X}_{\mathbf{o i}}\right)\right\}
$$

The handoff probability for a specific user depends on the number of users $i$ that share the same channel. Thus, handoff probability is defined in terms of the system steady state probabilities as: 


$$
P_{H}=\sum_{\{\mathbf{k} \in \Omega\}} \pi_{\mathbf{K}} \sum_{i=1}^{R} \frac{i\left(k_{i}-k_{i+1}\right)}{\sum_{i=1}^{R} k_{i}} \frac{\eta}{\eta+\mu+\gamma_{i}}
$$

\section{Call interruption probability}

Call interruption probability is defined as the probability that a call is forced to terminate due to the interference and it depends on the number of users $i$ sharing the same channel within the analyzed cell. It is mathematically expressed as

$$
P_{I i}=\mathrm{P}\left\{\mathbf{X}_{\mathbf{o i}}<\min \left(\mathbf{X}_{\mathbf{s}}, \mathbf{X}_{\mathbf{r}}\right)\right\}
$$

Thus, call interruption probability can be calculated as:

$$
P_{I}=\sum_{\{\mathbf{K} \in \Omega\}} \pi_{\mathbf{K}} \sum_{i=1}^{R} \frac{i\left(k_{i}-k_{i+1}\right)}{\sum_{i=1}^{R} k_{i}} \frac{\gamma_{i}}{\eta+\mu+\gamma_{i}}
$$

\section{Probability of Call Forced Termination}

Call forced termination probability (Pft) is considered as one of the most important QoS metrics for RP-based mobile cellular systems (Heredia-Ureta et.al., 2003), (Jiang \& Rappaport, 2004). In particular, in SDMA cellular systems, call forced termination may result from either handoff failure or link unreliability due to the excessive co-channel interference. Since a combinatorial analysis in order to evaluate call forced termination probability is very complex, a signal flow diagram is used to characterize the call states transitions ), (Jiang \& Rappaport, 2004), (Robichaud et.al, 1962).

Figure 4 shows the signal flow diagram for an SDMA cellular system with the capability of replicate a basic channel up to three times. Thus, the signal flow diagram is solved by considering that the call initiation state is the source node and the forced call termination is the sink node, and then by using the Mason's rule. Call state transitions include handoff attempts, successful call termination and interruption probability.

\section{Performance evaluation of mobile SDMA cellular systems}

In this section, the impact of mobility and radio environment in SDMA cellular networks is evaluated by means of teletraffic analysis considering the teletraffic model presented in the previous section. Blocking and Call Forced Termination probabilities are evaluated and compared when Duplicate at First and Duplicate at Last admission policies are used. Numerical evaluations are conducted considering that the system has $C=10$ basic channels that can replicated up to three times. Offered traffic is varied from a $=10$ to 50 Erlangs/cell. In addition, Blocking Calls Cleared (BCC) policy is considered. Mean service time $\mathbf{E}\left\{\mathbf{X}_{\mathrm{s}}\right\}=$ $180 \mathrm{~s}$. In order to evaluate mobility mean cell dwell time is varied $\left(\mathbf{E}\left\{\boldsymbol{X}_{\mathrm{r}}\right\}\right)$ and to evaluate radio environment mean beam overlapping time $\left(\mathbf{E}\left\{\mathbf{X}_{\mathrm{o} i}\right\}\right)$ is varied.

\subsection{The impact of mobility in SDMA cellular systems}

Figures 5-8 show the impact of mobility in blocking and call forced termination probabilities for different scenarios. Mean cell dwell time is varied $\left(\mathbf{E}\left\{\mathbf{X}_{\mathrm{r}}\right\}=1000,5000\right.$, No mobility) Evaluations presented in this section do not consider link unreliability due to the excessive co-channel interference. 


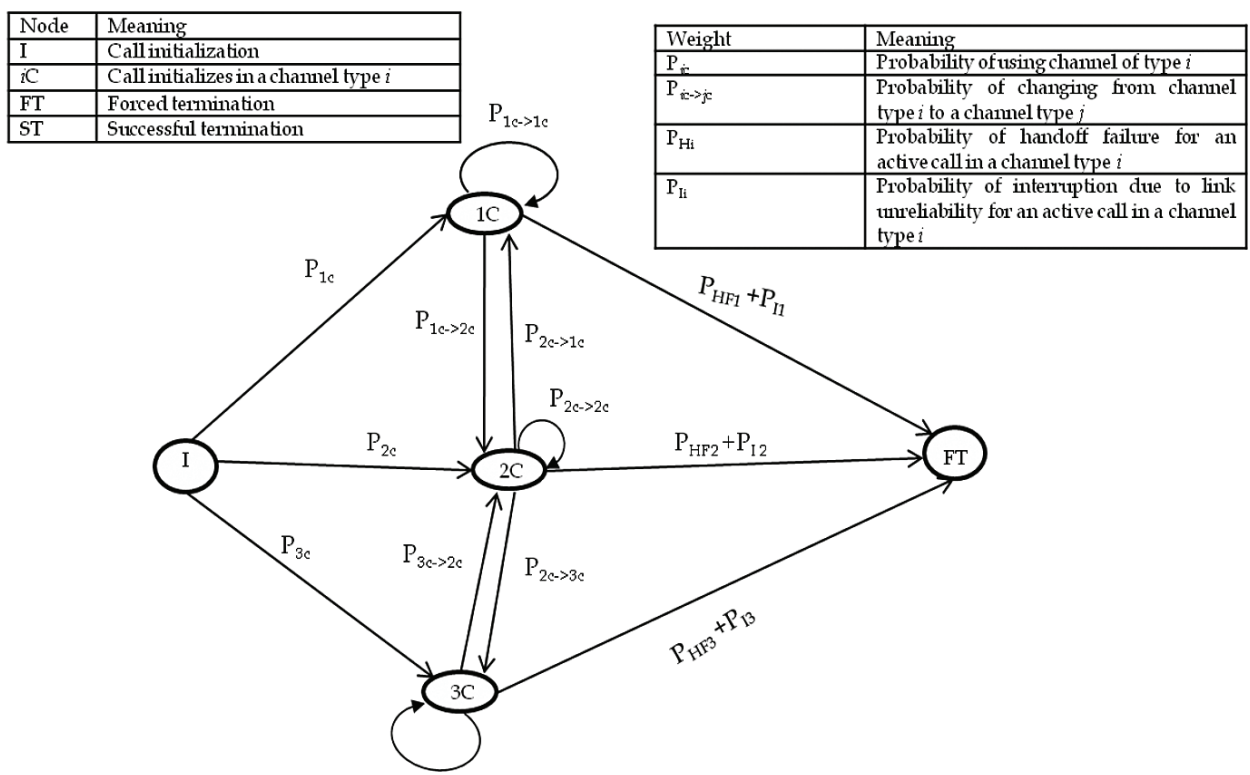

Fig. 4. SDMA system with Duplicate at First Policy .

From figures 5-6 it is possible to observe that the blocking probability is almost insensible to the residence time and to the call admission control policy. However, from figures 7-8 it is possible to observe that call forced termination probability is very sensible to the mobility. As the mean residence time decreases, call forced termination probability increases exponentially. This is because of the handoff probability also increases. Notice that when no

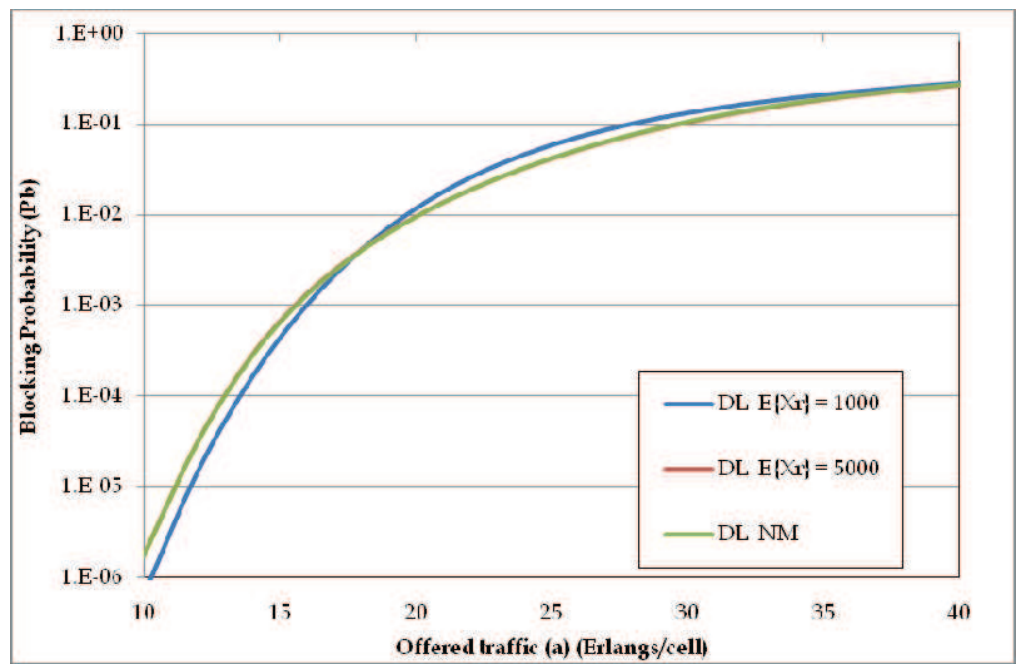

Fig. 5. Blocking Probability for a system with Duplicate at Last Policy. No link unreliability is considered. 


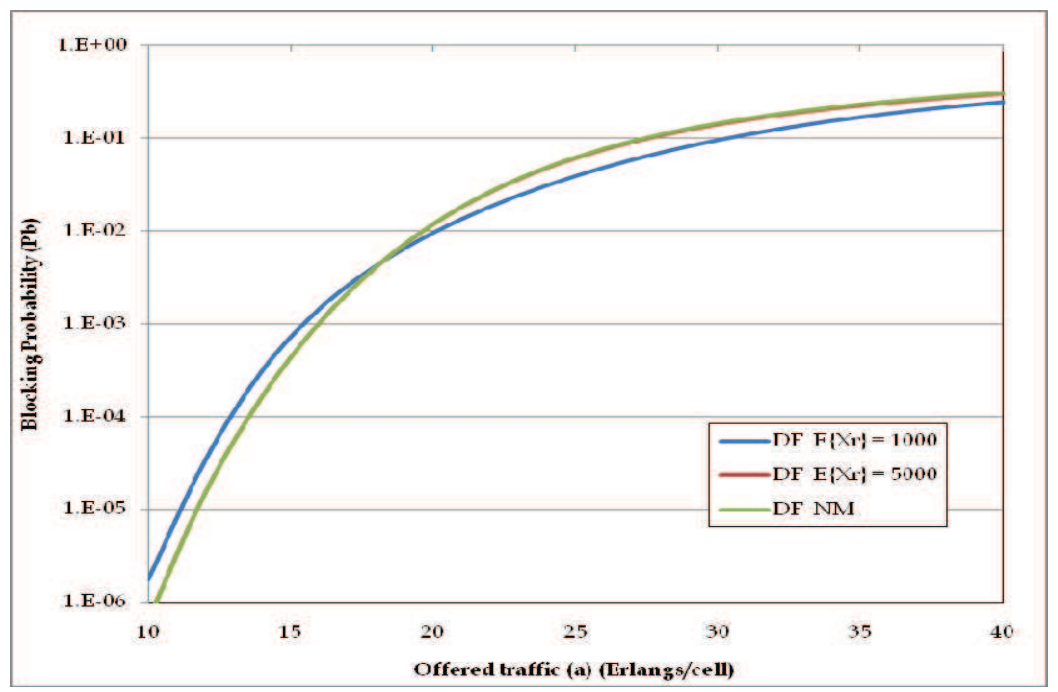

Fig. 6. Blocking Probability for a system with Duplicate at First Policy . No link unreliability is considered.

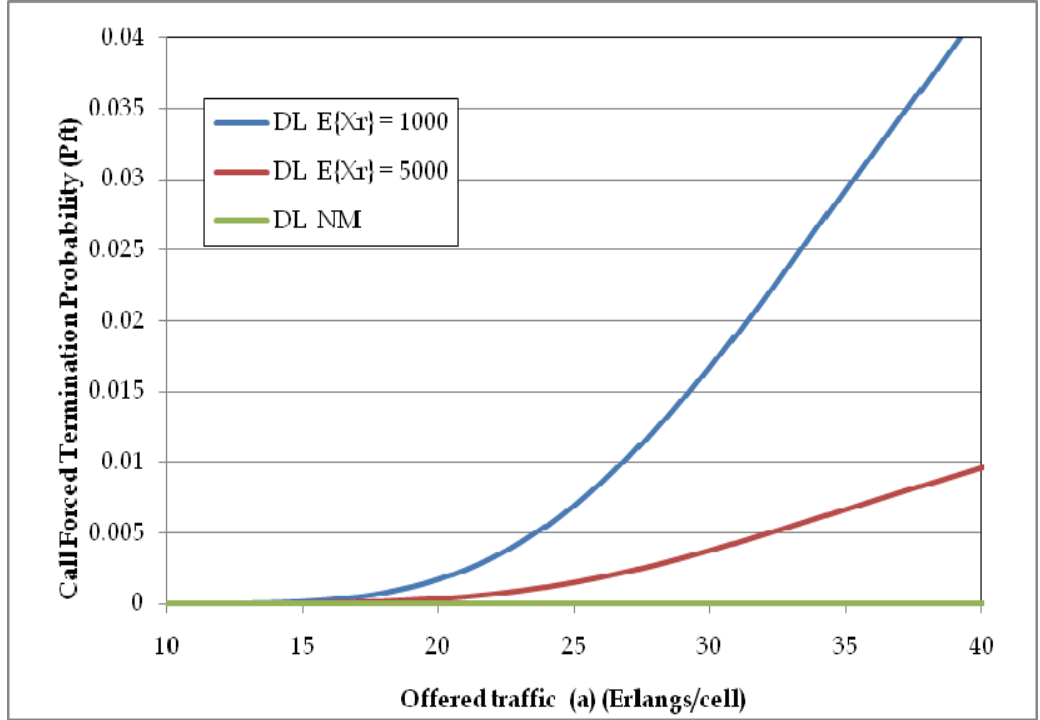

Fig. 7. Call Forced Termination Probability for a system with Duplicate at Last Policy. No link unreliability is considered. 


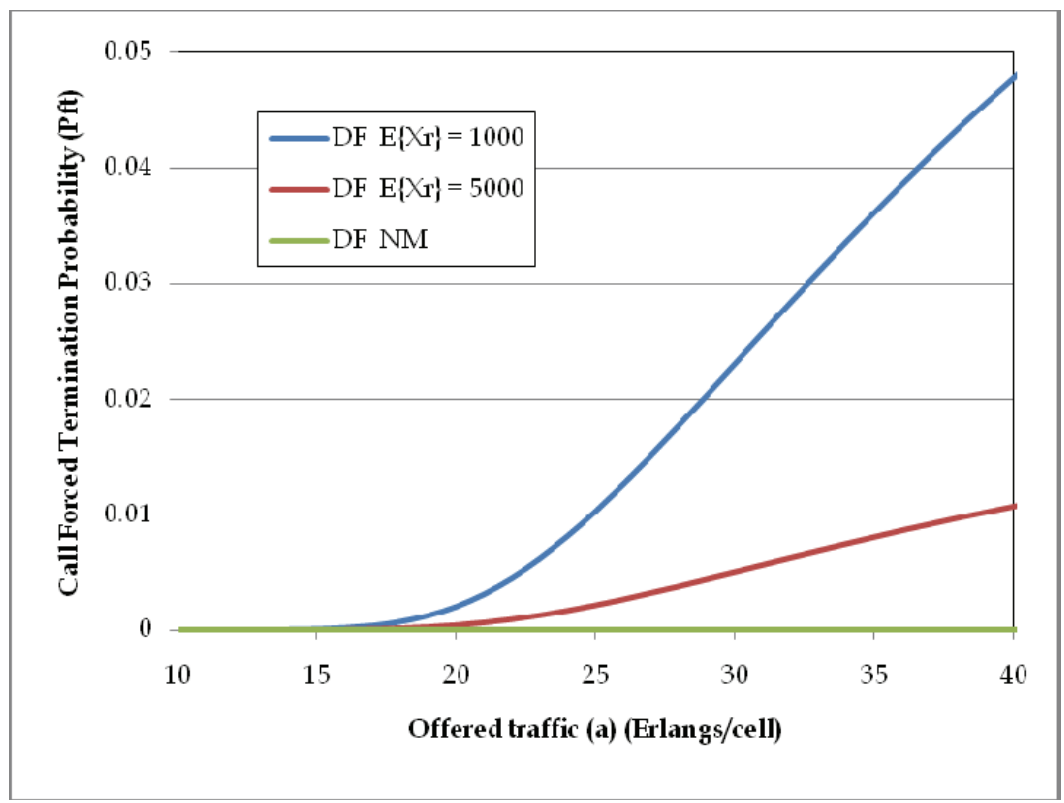

Fig. 8. Call Forced Termination Probability for a system with Duplicate at First Policy. No link unreliability is considered.

mobility is considered call forced termination is zero for all cases. This is because there are no causes of forced termination. Figures 7-8 show that the "Duplicate at First" policy is more sensible to the mobility. This is because in the scenario where there is more mobility, there are also more handoff requests.

\subsection{The impact of radio environment in SDMA cellular systems}

Figures 9-12 show the impact of radio environment in blocking and call forced termination probabilities for different scenarios. Mean beam overlapping time $\left(\mathbf{E}\left\{\mathbf{X}_{\mathrm{oi}}\right\}=4000,8000\right.$, No link unreliability). Evaluations presented in this section do not consider link unreliability due to the excessive co-channel interference.

Figures 9-12 show how the link unreliability due to the co-channel interference brought within the cell because of the intra-cell reuse affects the system's performance. Notice that the larger beam overlapping time represents the scenario where the channel conditions are better, that is where Signal to Interference Ratio is not very affected due to the intra-cell reuse

From figures 9-12 it is possible to observe that "Duplicate at Last" policy provides the best performance in terms of call forced termination probability. This behaviour is because the more mobility the more interference is carried within the cell.

\section{Conclusions}

In this chapter an outline of the smart antenna technology in mobile cellular systems was given. An historical overview of the development of smart antenna technology was 


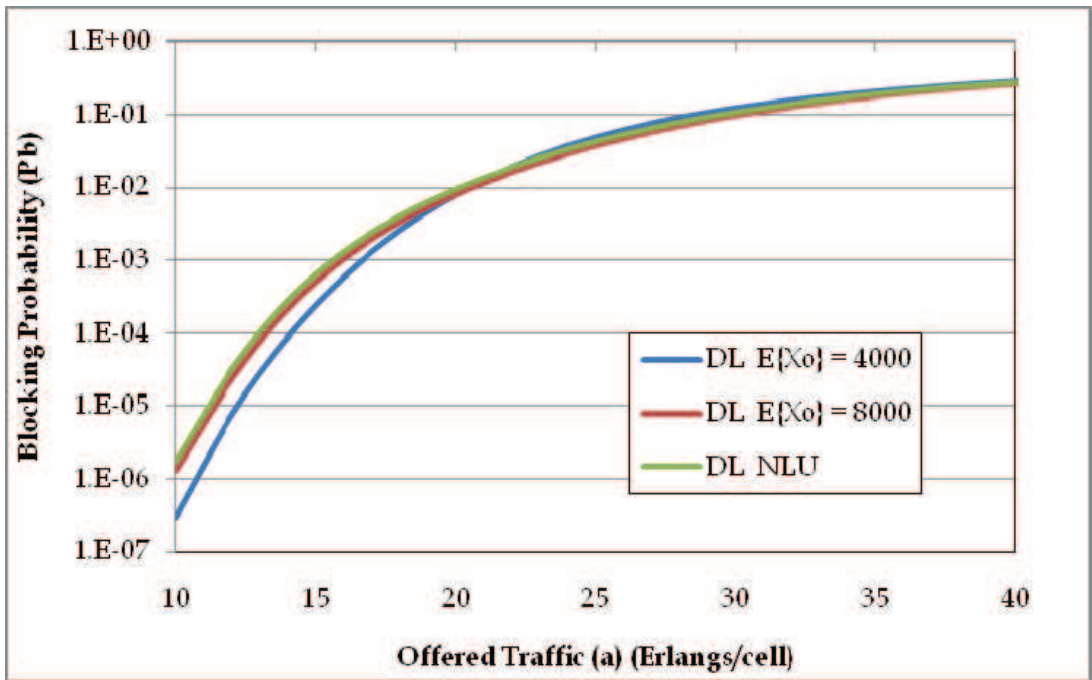

Fig. 9. Blocking Probability for a system with Duplicate at Last Policy. No mobility is considered

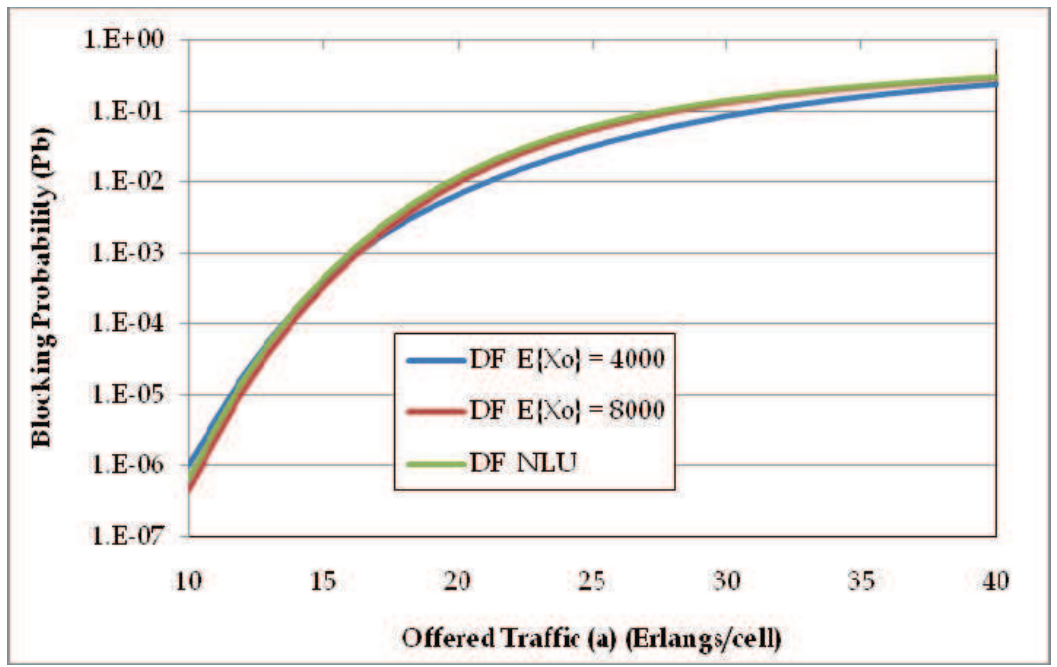

Fig. 10. Blocking Probability for a system with Duplicate at First Policy. No mobility is considered 


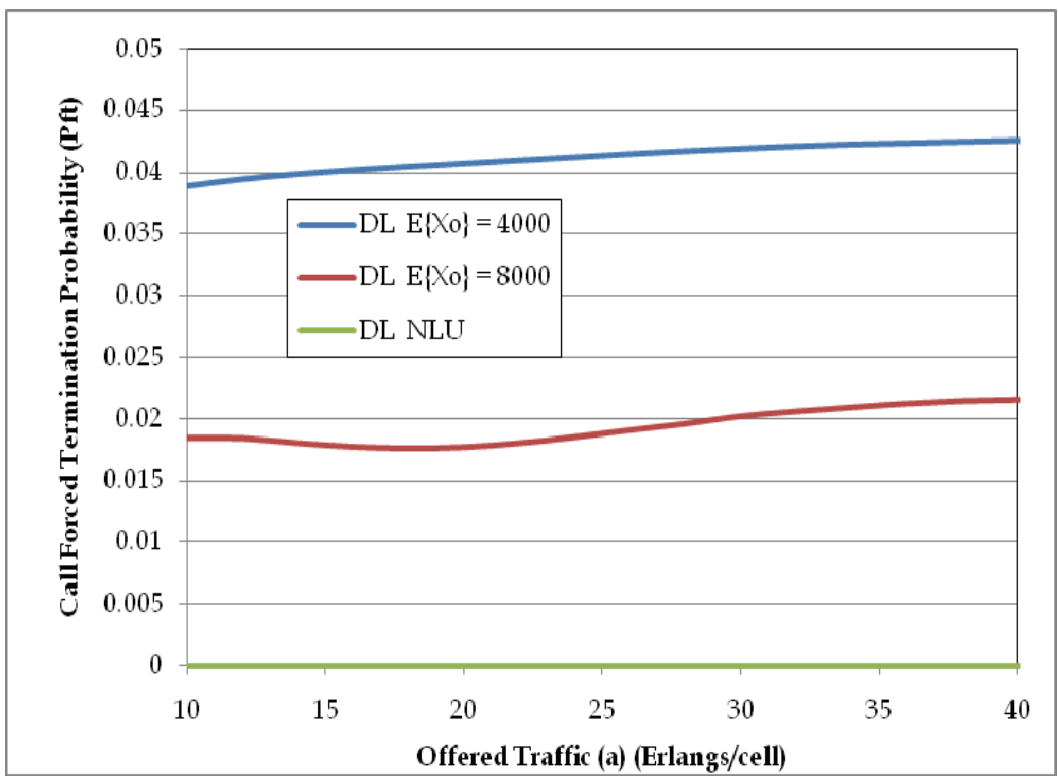

Fig. 11. Call Forced Termination Probability for a system with Duplicate at Last Policy. No mobility is considered

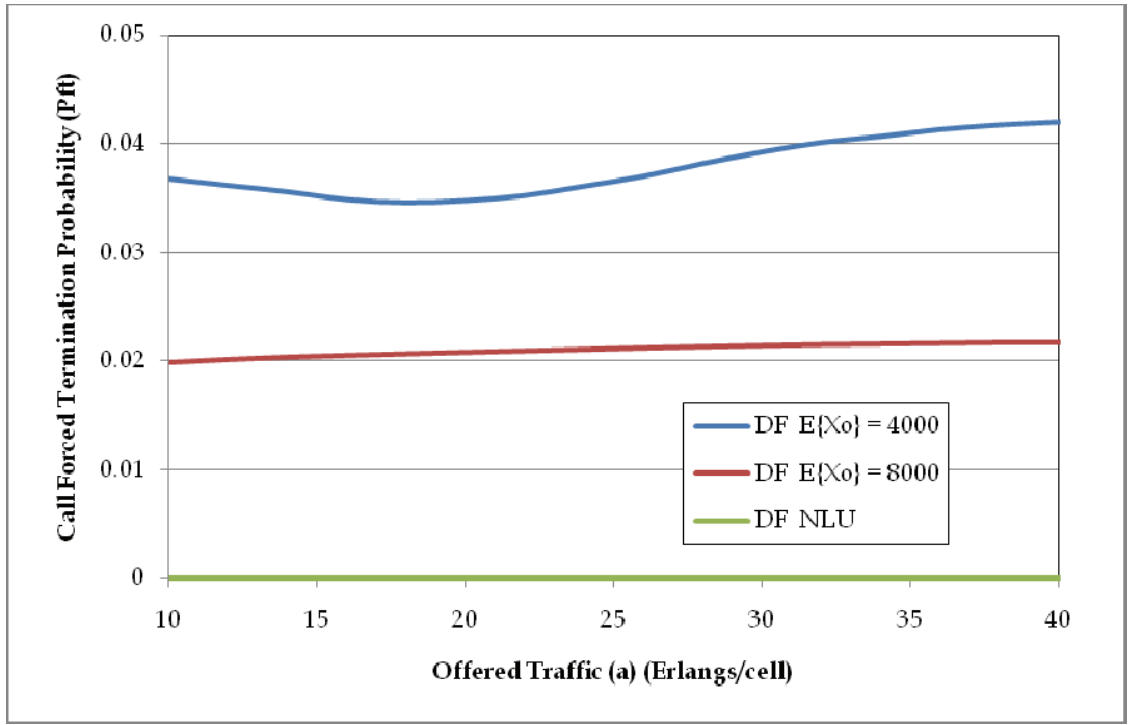

Fig. 12. Call Forced Termination Probability for a system with Duplicate at First Policy. No mobility is considered 
presented. Main aspects of the smart antenna components (array antenna and signal processing) were described. Main configurations and applications in cellular systems were summarized and some commercial products were addressed.

Spatial Division Multiple Access was emphasized because it is the technology that is considered the last frontier in spatial processing to achieve an important capacity improvement. Critical aspects of SDMA system level modeling were studied. In particular, users' mobility and radio environment issues are considered. Moreover, the impact of these aspects in system's performance were evaluated through the use of a new proposed system level model which includes mobility as well as channel conditions. Blocking and call forced termination probability were used as QoS metrics.

\section{References}

3GPP TR 25.913, (2009), Requirements for Evolved UTRA (EUTRA) and Evolved UTRAN (E-UTRAN).

Alexiou, A.; Navarro, M. \& Heath, R.W., (2007), Smart Antennas for Next GenerationWireless Systems, EURASIP Journal on Wireless Communications and Networking, Hindawi Publishing Corporation, vol. 2007, Article ID 20427, 2 pages.

Allen, B. \& Ghavami, M., (2005), Adaptive Array Systems Fundamentals and Applications, John Wiley \& Sons Ltd, England.

Applebaum, S.P., (1976), Adaptive arrays. IEEE Transactions on Antennas and Propagation, vol. 24, pp. 585-598, September 1976.

Balanis, C.A. (2005), Antenna Theory Analysis and Design, Third Edition, John Wiley \& Sons, Inc., Hoboken, New Jersey, 2005.

Ball, C.F.; Mullner, R.; Lienhart, J. \& Winkler, H. (2009), Performance analysis of Closed and Open loop MIMO in LTE, Proceedings European Wireless Conference 2009 (EW 2009), pp. 260 - 265, Aalborg, Denmark, May 2009.

Bettstetter, C., (2003), Mobility modelling in wireless networks categorization, smooth movement, and border effects, Mobile Computing and Communications Review, vol. 5, no. 3, pp. 55-67, 2003.

Boggia, G.; Camarda, P.; D’Alconzo, A.; De Biasi, A.; \& Siviero, M. (2005). Drop call probability in established cellular networks: from data analysis to modelling, in Proceedings of IEEE Vehicular Technology Conference 2005 Spring (VTC Spring'05), pp. 2775-2779, Stockholm, Sweden, May-Jun. 2005.

Boukalov, A.O. \& Haggman, S.-G., (2000), System aspects of smart-antenna technology in cellular wireless communications-an overview, IEEE Transactions on Microwave Theory and Techniques, vol. 48, no. 6, pp. 919-1929, June 2000.

Butler J. \& Lowe R., (1961), Beam-forming matrix simplifies design of electronically scanned antennas, Electronic Devices, vol. 9, no. 8, pp. 1730-1733, Apr. 1961.

Cardieri, P. \& Rappaport, T., (2001), Channel allocation in SDMA cellular systems, in Proceedings of IEEE Vehicular Technology Conference Fall 2001 (VTC Fall'01), vol. 1, pp. 399-403, Atlantic City, New Jersey, USA October 2001.

Cheblus, E. \& Ludwin, W., (1995), Is handoff traffic really Poissionian?, in Proceedings of IEEE International Conference on Universal Personal Communications (ICUPC'95), pp. 348-353, Tokyo, Japan, November 1995. 
Chiani, M.; Win, M.Z. \& Hyundong Shin, (2010), MIMO Networks: The Effects of Interference, IEEE Transactions on Information Theory, vol. 56, no.1, pp. 336-349, January 2010.

Cooper, R.B, (1990), Introduction to Queueing Theory, Washington D.C. CEE Press Book, 1990.

Czylwik A. \& Dekorsky, A., (2001) System level simulations for downlink beamforming with different array topologies, in Proceedings of IEEE Global Communications Conference 2001 (GLOBECOM'01), vol. 5, pp. 3222-3226, San Antonio, Texas, USA, November 2001.

Derneryd, A. \& Johannisson, B., (1999), Adaptive base-station antenna arrays, Ericsson Review No. 3, vol.76, pp. 136-137, 1999, Online Available: http://www.ericsson.com/ericsson/corpinfo/publications/review/1999_03/files /1999033.pdf

Galvan-Tejada G.M. \& Gardiner, J.G., (2001) Theoretical model to determine the blocking probability for SDMA systems, IEEE Transactions on Vehicular Technology, Vol. 50, No. 4, pp. 1279-1288, September 2001.

Galvan-Tejada, G.M. \& Gardiner, J.G., (1999), Theoretical blocking probability for SDMA, Communications, Institute of Electrical Engineers Proceedings, vol. 146, no. 5, pp. 303306, October 1999.

Galvan-Tejada, G.M. \& Gardiner, J.G., (2001), Performance of a Wireless Local Loop System Based on SDMA for Different Propagation Conditions, in Proceedings of IEEE Global Communications Conference 2001 (GLOBECOM '01), vol. 6, pp. 3594-3598, San Antonio, Texas USA December 2001.

Gowrishankar, R.; Demirkol, M.F. \& Zhengquing, Y., (2005), Adaptive modulation for MIMO systems and throughput evaluation with realistic channel model, in Proceedings of International Conference on Wireless Networks, Communications and Mobile Computing 2005, vol. 2 pp 851 - 856, Cologne, Germany, August 2005.

Gross, F., (2005), Smart Antennas for Wireless Communications, with Matlab, Mc Graw Hill, USA, 2005.

Hammuda, H. (1997), Cellular Mobile Radio Systems, John Wiley \& Sons. England, 1997.

Hansen, R.C., (1998) Phased Array Antennas, Wiley, New York, 1998.

Hemrungrote, S.; Hori, T.; Fujimoto, M. \& Nishimori, K., (2010), Channel capacity characteristics of multi-user MIMO systems in urban area, in Proceedings of IEEE Antennas and Propagation Society International Symposium 2010 (APSURSI 2010), Toronto, Ontario, Canada, July 2010.

Heredia-Ureta, H; Cruz-Pérez, F.A. \& Ortigoza-Guerrero, L., (2003), Capacity optimization in multiservice mobile wireless networks with multiple fractional cannel reservation, IEEE Transactions on Vehicular Technology, vol. 52, no. 6, pp. 15191539, November 2003

Ho M.; Stuber, G.L. \& Austin, M.D., (1998), Performance of switched-beam smart antennas for cellular radio systems, IEEE Transactions on Vehicular Technology, vol. 47, no.1, pp. 10-19, February 1998.

Hoymann, C., (2006), MAC Layer Concepts to Support Space Division Multiple Access in OFDM based IEEE 802.16s, Wireless Personal Communications, p. 23, May 2006

$\mathrm{Hu}, \mathrm{H}$. \& Zhu, J, (2002), A Combined Beam Hopping and Single Beam Switched-Beam Smart Antennas Scheme and Its Performance Analysis, in Proceedings of IEEE 
Region 10 Conference on Computers, Communications, Control and Power Engineering 2002 (TENCON 2002), Beijing, China, October 2002.

iBurst, (2004), iBurst Broadband Wireless System Overview, ArrayComm, White paper, October 2004, Online Available:

http://www.arraycomm.com/docs/iBurstOverview.pdf

ITU-R M.1801, (2007), Radio interface standards for broadband wireless access systems, including mobile and nomadic applications, in the mobile service operating below $6 \mathrm{GHz}$

Jiang, H. \& Rappaport, S.S. (1994), Hand-off analysis for CBWL schemes in cellular communications, in Proceedings of International Conference of Universal Personal Communications 1994 (UPC 1994), pp. 496-500, San Diego, CA, USA October 1994

Jingming-Wang \& Daneshrad, B. (2005), A comparative study of MIMO detection algorithms for wideband spatial multiplexing systems, in Proceedings of IEEE Wireless Communications and Networking Conference 2005 (WCNC 2005), vol. 1, pp. 408 - 413, New Orleans, L.A., U.S.A. 2005.

Kaiser, T., (2005), When will Smart antennas be ready for the market?, IEEE Signal Processing Magazine, vol. 22, no. 2, pp. 87-92, March 2005.

Krim, H. \& Viberg, M., (1996), Two decades of array signal processing research: the parametric approach, IEEE Signal Processing Magazine, vol. 14, no. 4, pp. 67-94.

Kusume, K.; Dietl, G.; Abe, T.; Taoka, H. \& Nagata, S., (2010), System Level Performance of Downlink MU-MIMO Transmission for 3GPP LTE-Advanced, in Proceedings of Vehicular Technology Conference Spring 2010 (VTC Spring'10), Taipei, May 2010.

Liberti J.C. \& Rappaport T.S., (1999), Smart Antennas for Wireless Communications: IS-95 and Third Generation CDMA Applications, Prentice Hall PTR, Upper Saddle River, New Jersey, 1999.

Lin, Y.B.; Mohan, S.; \& Noerpel, A., (2004), Queuing priority channel assignment strategies for PCS and handoff initial access, IEEE Transactions on Vehicular Technologies, vol. 43. no. 3, pp. 704-712, August 1994.

Litva, (1996), Digital Beamforming in Wireless Communications, Artech House Mobile Communications Series,U.S.A 1996.

Liu, H. \& Zeng, Q.-A., (2004), Performance Analysis of Handoff Scheme in Mobile Radio Systems with Smart Antennas, in Proceedings of World Wireless Congress 2004 (WWC'04), San Francisco, California, USA, May 2004.

Liu, H.; Xu, Y. \& Zeng, Q.-A., (2005), Modeling and performance analysis of future generation multimedia wireless and mobile networks using smart antennas, in Proceedings of Wireless Communications and Networks Conference 2005 (WCNC'05), pp. 1286-1291, New Orleans, LA, USA, March 2005.

Mailloux, R.J., (1994), Phased Array Antenna Handbook, Artech House, Norwood, MA, 1994.

Nagai, Y. \& Kobayashi, T., (2005), Statistical characteristics of pedrestrians' motion and effects on teletraffic of mobile communication networks, in Proceedings of IEEE Wireless and Optical Communications Networks 2005 (WOCN'05), pp. 377-382, Dubai, United Arab Emirates, March 2005.

Nasri, R.; Kammoun, A.; Stephenne, A. \& Affes, S., (2008), System - Level Evaluation of a Downlink OFDM Kalman - Based Switched-Beam System with Subcarrier Allocation Strategies, in Proceedings of Vehicular Technology Conference Fall 2008 (VTC Fall'08), Calgary, Alberta, Canada, September, 2008. 
Ngamjanyaporn, P.; Phongcharoenpanich, C.; Akkaraekthalin, P. \& Krairiksh, M., (2005), Signal-to-interference ratio improvement by using a phased array antenna of switched-beam elements, IEEE Transactions on Antennas and Propagation, vol. 53, no. 5, pp. 1819-1828, May 2005.

Nishimori, K.; Kudo, R.; Takatoti, Y.; Ohta, A. \& Tsunekawa, K., (2006), Performance Evaluation of Multi-User MIMO-OFDM Testbed in an Actual Indoor Environment, in Proceedings of International Symposium on Personal, Indoor and Mobile Radio Communications 2006 (PIMRC 2006), Helsinki, Finland, September 2006.

Okamoto, G., (2003), Developments and advances in smart antennas for wireless communications, Santa Clara University, Tech. Rep., 2003, Online Available: www.wmrc.com/bussinessbriefing/pdf/wireless_2003/Publication/okamoto.pdf

Pabst, R.; Ellenbeck, J.; Schinnenburg, M. \& Hoymann, C., (2007) System level performance of cellular WIMAX IEEE 802.16 with SDMA-enhanced medium access, in Proceedings of IEEE Wireless Communications and Networking Conference 2007 (WCNC'07), pp. 1820-1825, Hong Kong, March 2007.

Parra, I.; Xu, G. \& Liu, H., (1995), A Least Squares Projective Constant Modulus Approach, in Proceedings of IEEE International Symposium on Personal, Indoor and Mobile Radio Communications 1995 (PIMRC 1995), vol. 2, pp. 673-676, Toronto, Canada, September 1995.

Pattan, B., (2000), Robust Modulation Methods and Smart Antennas in Wireless Communications, Prentice Hall, New York, 2000.

Paulraj, A.J. \& Papadias, C. B. (1997), Space-time processing for wireless communications, IEEE Signal Processing Magazine., vol. 14, no. 6, pp. 49-83, November 1997.

Peng, M. \& Wang, W., (2005), Comparison of capacity between adaptive tracking and switched beam smart antenna techniques in TDD-CDMA systems, in Proceedings of IEEE International Symposium on Microwave, Antenna, Propagation and EMC Technologies for Wireless Communications, 2005 (MAPE 2005), vol. 1, pp.135-139, Beijing, China, August 2005.

Phasouliotis, A. \& So, D.K.C., (2009), Performance Analysis and Comparison of Downlink MIMO MC-CDMA and MIMO OFDMA Systems, in Proceedings of Vehicular Technology Conference Spring 2009 (VTC Spring'09), Barcelona, Spain, April 2009.

Rajal, F., (2005), Why have smart antennas not yet gained traction with wireless network operators?, IEEE Antennas and Propagation Magazine, vol. 47, no. 6, pp. 124-126, December 2005.

Robichaud, L.P.A.; Boisvert, M. \& Robert, J., (1962), Signal Flow Graphs and Applications, Prentice Hall, 1962.

Rodríguez-Estrello, C.B. \& Cruz-Pérez, F.A., (2009), System Level Analytical Model for SDMA Mobile Cellular Networks Considering Cochannel Interference, Proceeding of IEEE International Symposium on Personal, Indoor Mobile Radio Communications 2009, (PIMRC 2009), September, Tokyo, Japan 2009

Rodríguez-Estrello, C.B.; Hernández-Valdéz, G. \& Cruz-Pérez F.A., (2009), System Level Analysis of Mobile Cellular Networks Considering Link Unreliability, IEEE Transactions on Vehicular Technology, vol. 58, no.2, February 2009

Schmidt, R. O., (1979), Multiple Emitter Location and Signal Parameter Estimation, in Proceedings of RADC Spectrum Estimation Workshop 1979, Griffiss AFB, New York, pp. 243-258, 1979 
Schweppe, F., (1968), Sensor-array data processing for multiple-signal sources, IEEE Transactions on Information Theory, vol. 14, no. 2, pp. 294 - 305, March 1968

Seki, H. \& Tsutsui, M., (2007), Throughput Performance of Pre-coding MIMO Transmission with Multi-Beam Selection, in Proceedings of IEEE International Conference on Communications 2007 (ICC '07), pp 2785-2790, Glasgow, Scotland, June 2007.

SentieM ${ }^{\mathrm{TM}}$, (2009), SentieM ${ }^{\mathrm{TM}}$ Technologies Enhance Coverage, Capacity and Throughput, the Fundamentals for a Superior Mobile WiMAX ${ }^{\mathrm{TM}}$ Network, Alvarion, Technology White Paper, July 2009, Online Available:

http://alvarion.net/images/stories/resourcecenter/whitepapers/Mobile_WiMAX _Tech_Edge_wp_LR_1_.216.pdf

Shim, D \& Choi, S., (1998), Should the smart antenna be a tracking beam array or switching beam array?, in Proceedings of IEEE Vehicular Technology Conference 1998 (VTC'98), vol. 1, pp. 494-498, Ottawa, Ontario, Canada, May 1998.

Shuangmei, C; Jianhua, L; Ying, Y. \& Zehong, X., (2004), A novel method for performance analysis of SDMA, in Proceedings of IEEE Vehicular Technology Conference Fall 2004 (VTC Fall'04), vol. 6, pp. 4180-4184, Los Angeles, California, USA, September 2004.

Tangeman, M., (2004), Influence of the user mobility on the spatial multiplex gain of an adaptive SDMA system, in Proceedings of Personal Indoor Mobile Radio Communications 2004 (PIMRC'94), vol. 2, pp. 745-749, Hague, Holland, September 2004.

Toşa, F., (2010), Comparisons of beamforming techniques for $4 \mathrm{G}$ wireless communications systems, Proceeding of IEEE $8^{\text {th }}$ International Conference on Communications (COMM 2010), Bucarest, Romania, June 2010.

Tsoulos, G.V., (1999), Smart antennas for mobile communication systems: Benefits and challenges, IEEE Communications Journal, vol. 11, no. 2, pp. 84-94, Apr. 1999.

Tsoulos, G.V.; Beach, M. \& McGeehan, J., (1997), Wireless Personal Communications for the 2 1st Century, European Technological Advances in Adaptive Antennas, vol. 35 no. 9, pp 102-109, September 97

Van Atta, L. C., (1959), Electromagnetic Reflector, U.S. Patent 2908 002, Serial no. 514 040, October 1959.

Van Rooyen, P. (2002), Advances in space-time processing techniques open up mobile apps, November, 2002, EE|Times The global electronics engineering community News $\mathcal{E}$ Analysis, Online Available:

http:/ / www.eetimes.com/electronics-news/4143480/Advances-in-space-timeprocessing-techniques-open-up-mobile-apps.

Widrow, B.; Mantey, P.E.; Griffiths, L.J. \& Goode, B. B., (1967), Adaptive Antenna systems, in Proceedings of the IEEE, vol. 55, no. 12, December 1967.

Zhongding, L; Chin, F.P.S. \& Ying-Chang, L, (2005), Orthogonal switched beams for downlink diversity transmission, IEEE Transactions on Antennas and Propagation, vol. 52, no.7, pp. 2169-2177, July 2005.

Ziskind, I. \& Wax, M., (1988), Maximum Likelihood Localization of Multiple Sources by Alternating Projection, IEEE Transactions on Acoustics, Speech, and Signal Process., vol. 36, no. 10, pp.1553-1560, October 1988.

Zonoozi, M.M. \& Dassanayake, P., (1997), User mobility modeling and characterization of mobility patterns, IEEE Journal on Selected Areas in Communications, vol. 15, no. 7, pp. 1239-1252, September 1997. 


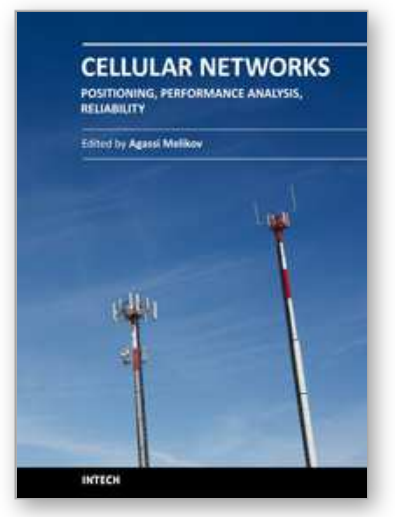

\author{
Cellular Networks - Positioning, Performance Analysis, Reliability \\ Edited by Dr. Agassi Melikov
}

ISBN 978-953-307-246-3

Hard cover, 404 pages

Publisher InTech

Published online 26, April, 2011

Published in print edition April, 2011

Wireless cellular networks are an integral part of modern telecommunication systems. Today it is hard to imagine our life without the use of such networks. Nevertheless, the development, implementation and operation of these networks require engineers and scientists to address a number of interrelated problems. Among them are the problem of choosing the proper geometric shape and dimensions of cells based on geographical location, finding the optimal location of cell base station, selection the scheme dividing the total net bandwidth between its cells, organization of the handover of a call between cells, information security and network reliability, and many others. The book focuses on three types of problems from the above list Positioning, Performance Analysis and Reliability. It contains three sections. The Section 1 is devoted to problems of Positioning and contains five chapters. The Section 2 contains eight Chapters which are devoted to quality of service (QoS) metrics analysis of wireless cellular networks. The Section 3 contains two Chapters and deal with reliability issues of wireless cellular networks. The book will be useful to researches in academia and industry and also to post-gradute students in telecommunication specialitiies.

\title{
How to reference
}

In order to correctly reference this scholarly work, feel free to copy and paste the following:

Carmen B. Rodríguez-Estrello and Felipe A. Cruz Pérez (2011). An Insight into the Use of Smart Antennas in Mobile Cellular Networks, Cellular Networks - Positioning, Performance Analysis, Reliability, Dr. Agassi Melikov (Ed.), ISBN: 978-953-307-246-3, InTech, Available from: http://www.intechopen.com/books/cellular-networkspositioning-performance-analysis-reliability/an-insight-into-the-use-of-smart-antennas-in-mobile-cellularnetworks

\section{INTECH}

open science | open minds

\section{InTech Europe}

University Campus STeP Ri

Slavka Krautzeka 83/A

51000 Rijeka, Croatia

Phone: +385 (51) 770447

Fax: +385 (51) 686166

www.intechopen.com
InTech China

Unit 405, Office Block, Hotel Equatorial Shanghai

No.65, Yan An Road (West), Shanghai, 200040, China

中国上海市延安西路 65 号上海国际贵都大饭店办公楼 405 单元

Phone: +86-21-62489820

Fax: $+86-21-62489821$ 
(C) 2011 The Author(s). Licensee IntechOpen. This chapter is distributed under the terms of the Creative Commons Attribution-NonCommercialShareAlike-3.0 License, which permits use, distribution and reproduction for non-commercial purposes, provided the original is properly cited and derivative works building on this content are distributed under the same license. 